\title{
Automatic Selection of Sequence Transformations
}

\author{
By J. P. Delahaye
}

\begin{abstract}
Some methods of automatic selection of sequence transformations for accelerating the convergence of sequences are presented. Two theorems show the efficiency of these methods. This is confirmed by numerical experiments.
\end{abstract}

Introduction. When faced with the great number of various methods for accelerating the convergence of sequences [3], [4], [5], and also with the problem of the choice of parameters for some of them (Richardson process [8], $\rho$-algorithm [2]), the user is in a quite difficult position. If some precise information about the behavior of the sequence to be accelerated is known, it is possible to determine the most powerful acceleration method, but, even in this case, several possibilities remain. The user can (if he has plenty of time!) try all the methods and choose the best one with the help of some test problems.

In practice, however, it happens very often that the choice is arbitrary and thus is not the best one for the problem.

In this paper some automatic processes for selecting a good method of acceleration among several sequence transformations are proposed. All these processes work with the following scheme:

At the $n$th step the various transformations "in competition", $A_{1}, A_{2}, \ldots, A_{k}$, are applied to the sequence $\left(S_{n}\right)$ of a metric space $E$. The transformed points $A_{1}^{(n)}, A_{2}^{(n)}, \ldots, A_{k}^{(n)}$ are obtained. Then, one of the transformed points $A_{1}^{(n)}, A_{2}^{(n)}, \ldots, A_{k}^{(n)}$ is selected by using various tests (several are defined and studied). This point must be the best one at this step.

The use of these automatic selection methods is more expensive than the use of a single transformation for accelerating the convergence because it is necessary to use, simultaneously, several transformations. However, this is not a drawback because acceleration transformations are generally not expensive.

In the first section we present various general methods for selection, and we give some examples. In the second section we prove two theorems which legitimize the proposed methods. The numerical results of the third section confirm the practical efficiency of these methods.

\section{General Definitions for Selection Methods.}

1.1. Count Coefficients. Let $\mathfrak{R}_{i}^{(n)}$ be a relation (true or false depending on $i$ and $n$ ) defined for all $i \in\{1,2, \ldots, k\}$ and for all $n \in \mathbf{N}, n>n_{0}$.

Received August 1, 1980; revised November 25, 1980.

1980 Mathematics Subject Classification. Primary 65B99. 
Let us set the following count coefficients:

${ }^{0} r_{i}^{(n)}=\mid \begin{array}{ll}0 & \text { if } n<n_{0} \text { or if } \Re_{i}^{(n)} \text { is not true, } \\ 1 & \text { if } n \geqslant n_{0} \text { and if } \mathcal{R}_{i}^{(n)} \text { is true, }\end{array}$

${ }^{1} r_{i}^{(n)}=\operatorname{card}\left\{q \in\{0,1, \ldots, n\} \mid n \geqslant n_{0}\right.$ and $\mathscr{R}_{i}^{(n)}$ is true $\}$,

${ }^{2} r_{i}^{(n)}=\mid \begin{aligned} & 0 \quad \text { if } n<n_{0} \text { or if } \mathcal{R}_{i}^{(n)} \text { is not true, } \\ & \max \left\{q \in\left\{1,2, \ldots, n-n_{0}+1\right\} \mid\right. \\ & \left.\quad \mathcal{R}_{i}^{(n)}, \mathcal{R}_{i}^{(n-1)}, \ldots, \Re_{i}^{(n-q+1)} \text { are true }\right\}, \text { if } n>n_{0} \text { and if } \mathcal{R}_{i}^{(n)} \text { is true. }\end{aligned}$

Note that, even if $\mathscr{R}_{i}^{(n)}$ is not defined, the count coefficients exist when $n<n_{0}$ and become zero. We have the following properties:

$$
\begin{gathered}
\forall i \in\{1,2, \ldots, k\}, \forall n \in \mathrm{N}: \\
{ }^{1} r_{i}^{(n)}=\sum_{j=0}^{n}{ }^{0} r_{i}^{(n)} ; \quad 0<{ }^{0} r_{i}^{(n)}<{ }^{2} r_{i}^{(n)} \leqslant{ }^{1} r_{i}^{(n)} ; n \geqslant n_{0} \Rightarrow{ }^{1} r_{i}^{(n)}<n-n_{0}+1 .
\end{gathered}
$$

It is possible to define other count coefficients. For example, a coefficient can be defined such that $\Re_{i}^{(n)}$ has more importance than $\Re_{i}^{(n-1)}, \mathscr{R}_{i}^{(n-1)}$ has more importance than $\mathcal{R}_{i}^{(n-2)}$ etc. Let $A_{1}, A_{2}, \ldots, A_{k}$ be $k$ sequence transformations. We denote by $A_{1}^{(n)}\left(S_{m}\right), A_{2}^{(n)}\left(S_{m}\right), \ldots, A_{k}^{(n)}\left(S_{m}\right)$, respectively, the sequences obtained by applying these transformations to the sequence $\left(S_{m}\right)$. For convenience, we shall also write $A_{i}^{(n)}$ instead of $A_{i}^{(n)}\left(S_{m}\right)$. All the sequences in this paper are sequences of a metric space $(E, d)$. If we substitute the relation $\Re_{i}^{(n)}$ for the relation $\left(\bigodot_{i}^{(n)}\right)$ : $A_{i}^{(n)}=A_{i}^{(n-1)}$ (which is defined only if $n>1$ ), we obtain other count coefficients denoted by ${ }^{0} c_{i}^{(n)},{ }^{1} c_{i}^{(n)},{ }^{2} c_{i}^{(n)}$.

Let $l \in \mathbf{N}$. If we substitute the relation $\mathcal{R}_{i}^{(n)}$ for the relation

$$
\left({ }_{l} \mathcal{D}_{i}^{(n)}\right): \max _{0<q<l} d\left(A_{i}^{n-q}, A_{i}^{n-q-1}\right)=\min _{1<j<k} \max _{0<q<l} d\left(A_{j}^{(n-q)}, A_{j}^{(n-q-1)}\right)
$$

(which is defined only if $n \geqslant l+1$ ), we obtain other count coefficients denoted by ${ }_{l}^{0} d_{i}^{(n)},{ }_{l}^{1} d_{i}^{(n)},{ }_{l}^{2} d_{i}^{(n)}$. When $l=0$, we denote by $\mathscr{D}_{i}^{(n)}$ the relation, $\mathcal{D D}_{i}^{(n)}$ and by ${ }^{0} d_{i}^{(n)},{ }^{1} d_{i}^{(n)}$, ${ }^{2} d_{i}^{(n)}$ the associated count coefficients.

Remark. The theorems proved in the following sections concern only the relations $\mathcal{C}_{i}^{(n)}$ and $\mathscr{D}_{i}^{(n)}$. However, it is possible to define other relations.

In particular, for methods based on interpolation (Richardson process [8], $\rho$-algorithm [4], etc.), we can use the relation

$$
d\left(S_{n}, S_{n}^{i}\right)=\min _{1<j<k} d\left(S_{n}, S_{n}^{j}\right),
$$

where $S_{n}^{j}$ is the value at $x_{n}^{j}$ of the interpolation function which takes the values $S_{n-k}, S_{n-k+1}, \ldots, S_{n-1}$ at $x_{n-k}^{j}, x_{n-k+1}^{j}, \ldots, x_{n-1}^{j}\left(\left(x_{n}^{1}\right),\left(x_{n}^{2}\right), \ldots,\left(x_{n}^{k}\right)\right.$ are $k$ sequences of parameters; for example, in the Richardson process it is assumed that: $\forall m, n: n \neq m \Rightarrow x_{n}^{j} \neq x_{m}^{j}$, and $\lim _{n \rightarrow \infty} x_{n}^{j}=0$ ). Such methods are more expensive than the methods studied in this paper, because the calculation of $S_{n}^{j}$ practically implies the determination of the interpolation function. However, some of the obtained results may be of interest [7].

1.2. Selection Methods. Let $f \in\{0,1,2\}$. With the help of the coefficients ${ }^{f_{i}}{ }^{(n)}$, we define a new transformation $A$ which selects at each step, one of the transformations $A_{i}(i \in\{1,2, \ldots, k\})$. 
Step $n$ of the Transformation $A={ }^{f} R\left(A_{1}, A_{2}, \ldots, A_{k}\right)$. We compute ${ }^{f_{1}(n)}$, ${ }^{f} r_{2}^{(n)}, \ldots,{ }^{f_{r}(n)}$. Let $i(n)$ be the smallest $i$ such that

$$
{ }^{r_{i}}(n)=\max _{1<j<k}{ }^{f_{j}(n)}
$$

We choose $A^{(n)}=A_{i(n)}^{(n)}$.

When applied together with the above count coefficients and $k$ sequence transformations, this scheme defines new sequence transformations denoted by ${ }^{0} C\left(A_{1}, A_{2}, \ldots, A_{k}\right),{ }^{1} C\left(A_{1}, A_{2}, \ldots, A_{k}\right),{ }^{2} C\left(A_{1}, A_{2}, \ldots, A_{k}\right),{ }_{i}^{0} D\left(A_{1}, A_{2}, \ldots, A_{k}\right)$, ${ }_{l}^{1} D\left(A_{1}, A_{2}, \ldots, A_{k}\right),{ }_{l}^{2} D\left(A_{1}, A_{2}, \ldots, A_{k}\right)$. If $l=0$, the last three transformations are denoted by ${ }^{0} D\left(A_{1}, A_{2}, \ldots, A_{k}\right),{ }^{1} D\left(A_{1}, A_{2}, \ldots, A_{k}\right),{ }^{2} D\left(A_{1}, A_{2}, \ldots, A_{k}\right)$.

Remark 1. Sometimes it is possible to compute recursively the count coefficients. For example, the computation of ${ }^{1} c_{i}^{(n)}$ need $A_{i}^{(0)}, A_{i}^{(1)}, \ldots, A_{i}^{(n)}$; but, in fact, it is possible to do it only with ${ }^{1} c_{i}^{(n-1)}, A_{i}^{(n)}, A_{i}^{(n-1)}$, because of the obvious relations:

$$
\begin{array}{ll}
{ }^{1} c_{i}^{(n)}={ }^{1} c_{i}^{(n-1)} & \text { if } A_{i}^{(n)} \neq A_{i}^{(n-1)}, \\
{ }^{1} c_{i}^{(n)}={ }^{1} c_{i}^{(n-1)}+1 & \text { if } A_{i}^{(n)}=A_{i}^{(n-1)} .
\end{array}
$$

Remark 2. In a selection method of sequence transformations, it is desirable to have a normalization, i.e. an eventual shift of the index such that the computation of $A_{i}^{(n)}$ uses, at each step, the same points of the sequence $\left(S_{n}\right)$; for example, points among $S_{0}, S_{1}, \ldots, S_{n}$. The calculation of $\varepsilon_{2}^{(n)}$ uses $S_{n}, S_{n+1}, S_{n+2}$ [3], hence, if $\varepsilon_{2}$ is one of the sequence transformations "in competition", we write $A_{i}^{(0)}=S_{0} ; A_{i}^{(1)}=$ $S_{1} ; A_{i}^{(n)}=\varepsilon_{2}^{(n-2)}$ for $n>2$.

When this normalization is respected, the new obtained sequence transformations are also normalized.

Remark 3. It is possible, in practical cases, that one of the used transformations can no longer be applied (for example in the case of division by zero). In such a case two kinds of strategies can be employed.

When this is the case for the transformation $A_{j}$ at the step $n_{0}$ :

$\left(1^{\circ}\right)$ For all $n>n_{0}$, only the transformations $A_{i}$ with $i \neq j$ are considered.

$\left(2^{\circ}\right)$ For all $n>n_{0}$, we take

$$
A_{j}^{(n)}=A_{j}^{\left(n_{0}\right)} ; \quad \mathcal{R}_{j}^{(n)} \Leftrightarrow \mathcal{R}_{j}^{\left(n_{0}\right)} .
$$

Remark 4. Methods of selection among a finite number of transformations are the only ones studied herein. However, it is possible to study the case of an infinite number of transformations. Such methods are studied in [7].

Example 1. Let $A_{1}, A_{2}$ be two given sequence transformations. ${ }^{0} D\left(A_{1}, A_{2}\right)$ is the sequence transformation $A$ which, at the step $n$, produces the more stable one of $A_{1}$ or $A_{2}$; i.e.

$$
\begin{array}{ll}
A^{(n)}=A_{1}^{(n)} & \text { if } d\left(A_{1}^{(n)}, A_{1}^{(n-1)}\right)<d\left(A_{2}^{(n)}, A_{2}^{(n-1)}\right), \\
A^{(n)}=A_{2}^{(n)} & \text { if } d\left(A_{2}^{(n)}, A_{2}^{(n-1)}\right)<d\left(A_{1}^{(n)}, A_{1}^{(n-1)}\right) .
\end{array}
$$

${ }^{1} C\left(A_{1}, A_{2}\right)$ is the sequence transformation $A$ which, at the step $n$, produces the less frequently changed one of $A_{1}$ or $A_{2}$; i.e. $-A^{(n)}=A_{1}^{(n)}$ if in the first $n$ steps $A_{1}^{(q)}=A_{1}^{(q-1)}$ is more frequently true than $A_{2}^{(q)}=A_{2}^{(q-1)} ; A^{(n)}=A_{2}^{(n)}$ if not.

Example 2. Selection among Richardson processes [3], [4], [8]. Let $\left(t_{n}\right)$ be a sequence of real numbers such that $\lim _{n \rightarrow \infty} t_{n}=0$ and $i \neq j \Rightarrow t_{i} \neq t_{j}$. The 
Richardson process with parameters $\left(t_{n}\right)$ is the calculation of $T_{k}^{(n)}=P_{k}^{(n)}(0)$, where $P_{k}^{(n)}$ is the unique polynomial such that

$$
\left\{\begin{array}{l}
P_{k}^{(n)}\left(t_{n}\right)=S_{n} ; \quad P_{k}^{(n)}\left(t_{n+1}\right)=S_{n+1} ; \ldots ; P_{k}^{(n)}\left(t_{n+k}\right)=S_{n+k}, \\
d^{0} P_{k}^{(n)} \leqslant k .
\end{array}\right.
$$

When $k$ is fixed, we obtain a sequence transformation. But usually it is interesting to consider the diagonal transformation $n \rightarrow T_{n}^{(n)}$. For example, with three sequences of parameters $\left(t_{n}\right),\left(x_{n}\right),\left(y_{n}\right)$, we obtain three diagonal transformations $\left(T_{n}^{(n)}\right),\left(X_{n}^{(n)}\right),\left(Y_{n}^{(n)}\right)$ which can be denoted by $A_{1}^{(n)}, A_{2}^{(n)}, A_{3}^{(n)}$. Below, we shall see that the new transformation ${ }^{1} D\left(A_{1}, A_{2}, A_{3}\right)$ is an interesting one, because it "pluralizes" the extrapolation properties of $A_{1}, A_{2}, A_{3}$. In such an example it is not necessary to "normalize" the sequence transformations $A_{1}, A_{2}, A_{3}$ because, at the step $n$, each of them uses the same points $S_{n}, S_{n+1}, \ldots, S_{2 n}$.

Example 3. Practically, the most interesting methods are the selections among transformations as various as possible. We shall see, in the third section, a few computational experiences with ${ }_{l}^{f} D\left(A_{1}, A_{2}, A_{3}, A_{4}, A_{5}, A_{6}\right)$ where

$A_{1}$ is the Richardson process [3], [8],

$A_{2}$ is the $\varepsilon$-algorithm [3], [10], [11],

$A_{3}$ is the $\rho$-algorithm [2],

$A_{4}$ is the Overholt process [3], [9],

$A_{5}$ is the $\theta$-algorithm [1], [3],

$A_{6}$ is the iterated $\Delta^{2}[4]$.

\section{Two Results on Selection Methods.}

2.1. Exact Transformations. We recall a few definitions which are used below. We say that the sequence transformation $A$ is regular for the family $\mathcal{S}$ of convergent sequences if

$$
\forall\left(S_{n}\right) \in \mathcal{S}: \lim _{n \rightarrow \infty} S_{n}=\lim _{n \rightarrow \infty} A^{(n)} .
$$

We say that the sequence transformation $A$ is semiregular for the family $\mathcal{S}$ of convergent sequences if

$$
\begin{aligned}
\forall\left(S_{n}\right) & \in \mathcal{S}\left[\exists n_{0} \in \mathrm{N}, \forall n>n_{0} A^{(n)}=A^{(n+1)}\right] \\
& \Rightarrow\left[\exists m_{0} \in \mathrm{N}, \forall m>m_{0} A^{(m)}=\lim _{n \rightarrow \infty} S_{n}\right] .
\end{aligned}
$$

We say that the sequence transformation $A$ is exact for the family $\mathcal{S}$ of convergent sequences if

We have

$$
\forall\left(S_{n}\right) \in \mathcal{S}, \exists m_{0} \in \mathbf{N}, \forall m>m_{0}: A^{(m)}=\lim _{n \rightarrow \infty} S_{n}
$$

$$
[A \text { exact }] \Rightarrow[A \text { regular }] \Rightarrow[A \text { semiregular }]
$$

Results concerning these properties are known for most of the acceleration methods ([3], [4], [6], etc.).

TheOREM 1. Let $\delta_{1}, \delta_{2}, \ldots, \delta_{k}$ be $k$ families of convergent sequences. For each $i \in\{1,2, \ldots, k\}$, let $A_{i}$ be a sequence transformation, exact for $\mathcal{S}_{i}$ and semiregular for $\delta_{1} \cup \delta_{2} \cup \cdots \cup \delta_{k}$. Then the transformations ${ }^{1} C\left(A_{1}, A_{2}, \ldots, A_{k}\right)$, ${ }^{2} C\left(A_{1}, A_{2}, \ldots, A_{k}\right),{ }_{l}^{1} D\left(A_{1}, A_{2}, \ldots, A_{k}\right),{ }_{l}^{2} D\left(A_{1}, A_{2}, \ldots, A_{k}\right)$ are exact for $\mathcal{S}_{1} \cup \mathcal{S}_{2}$ $\cup \cdots \cup \mathfrak{S}_{k}$. 
Proof. We indicate a proof only for ${ }^{1} C\left(A_{1}, A_{2}, \ldots, A_{k}\right)$ denoted by $A$. One can easily adapt this proof to the other cases.

Let $\left(S_{m}\right) \in \mathcal{S}_{1} \cup \mathcal{S}_{2} \cup \cdots \cup \mathcal{S}_{k}$. Let $I_{0}$ be the set of integers $i_{0}$ such that $A_{i_{0}}$ is exact for $\left(S_{m}\right)$. From the assumptions there is at least one element in $I_{0}$, but it is possible that there are several ones (because we have not supposed that the $\mathscr{S}_{i}$ are disjoint). Since $I_{0}$ is finite, there exists $p_{0} \in \mathbf{N}$ such that

$$
\forall i_{0} \in I_{0}, \forall p \geqslant p_{0}: A_{i_{0}}^{(p)}=S \quad\left(S=\lim _{m \rightarrow \infty} S_{m}\right) .
$$

Hence, for each $p \geqslant p_{0}, i_{0} \in I_{0}$, we have

$$
{ }^{1} c_{i_{0}}^{(p)} \geqslant p-p_{0} .
$$

Contrarily, if $i \notin I_{0}$, there exists an infinite set of integers $p$ such that

$$
A_{i}^{(p)} \neq A_{i}^{(p-1)}
$$

(this, from the assumption that $A$ is semiregular). Consequently, there exists an integer $p_{1} \geqslant p_{0}$ such that, for each $i \notin I_{0}, p \geqslant p_{1}$,

$$
{ }^{1} c_{i}^{(p)}<p-p_{0} .
$$

From (*), (**) we obtain that, for every $p \geqslant p_{1}, i(p) \in I_{0}$, and then $A^{(p)}=S$.

Remark 1 . Theorem 1 tells nothing about the selection methods ${ }^{0} C$ and ${ }_{l}^{0} D$, and it is possible, by counterexamples, to prove that generally the theorem is not true for ${ }^{0} \mathrm{C}$ and ${ }_{l}^{0} \mathrm{D}$.

Remark 2. The integer $p_{1}$ in the proof cannot be determined without other assumptions; but in practice $p_{1}$ is close to the index $p$ such that there exists $i$ : $A_{i}^{(p)}=S$.

Remark 3. Negative results concerning sequence transformations for accelerating the convergence show that the assumptions of semiregularity are essential [7]; without similar assumptions, no theorem such as Theorem 1 is possible.

2.2 Transformations for Accelerating the Convergence. We assume, in this section, that all sequences $\left(S_{n}\right)$ are convergent and verify

$$
\exists n_{0} \in \mathbf{N}, \forall n \geqslant n_{0}: S_{n} \neq \lim _{m \rightarrow \infty} S_{m} \text { and } S_{n} \neq S_{n+1} .
$$

We say that the sequence transformation $A$ accelerates the convergence of $\left(S_{n}\right)$ (resp. $\Delta$-accelerates) if

$$
\lim _{n \rightarrow \infty} d\left(A^{(n)}, \lim _{m \rightarrow \infty} S_{m}\right) / d\left(S_{n}, \lim _{m \rightarrow \infty} S_{m}\right)=0
$$

(respectively $\left.\lim _{n \rightarrow \infty} d\left(A^{(n+1)}, A^{(n)}\right) / d\left(S_{n+1}, S_{n}\right)=0\right)$. When $A$ accelerates (resp. $\Delta$-accelerates) all the sequences $\left(S_{n}\right)$ of the family $\mathcal{S}$, we say that $A$ accelerates $\mathcal{S}$ (resp. $\Delta$-accelerates $\mathcal{S}$ ). We say that the sequence transformation $A$ is fair for $\mathcal{S}$ if, for every sequence $\left(S_{n}\right) \in \mathcal{S}$,

$\left(F_{1}\right)$ either $A$ accelerates and $\Delta$-accelerates $\left(S_{n}\right)$,

$\left(F_{2}\right)$ or $\exists \varepsilon>0, \exists n_{0} \in \mathbf{N}, \forall n \geqslant n_{0} d\left(A^{(n+1)}, A^{(n)}\right) / d\left(S_{n+1}, S_{n}\right)>\varepsilon$.

When $E=\mathbf{R}$, a sufficient condition such that $A$ be fair for $\mathcal{S}$ is: all the sequences in $\delta$ are monotonic, and the sequence $\left(d\left(A^{(n+1)}, A^{(n)}\right) / d\left(S_{n+1}, S_{n}\right)\right)$ is always convergent to some limit $l$. The reason is that either $l=0$ and then $A$ accelerates and $\Delta$-accelerates (see [3]) or $l>0$ and then $\left(F_{2}\right)$ holds. 
Theorem 2. Let $l \in \mathbf{N}$. Let $A_{1}, A_{2}, \ldots, A_{k}$ be $k$ sequence transformations. For each $i \in\{1,2, \ldots, k\}$, we assume that $A_{i}$ accelerates and $\Delta$-accelerates $\mathcal{S}_{i}$, a family of convergent sequences, and that $A_{i}$ is fair for $\mathcal{S}_{1} \cup \mathcal{S}_{2} \cup \cdots \cup \mathcal{S}_{k}$. Then the transformations ${ }_{l}^{0} D\left(A_{1}, A_{2}, \ldots, A_{k}\right),{ }_{l}^{1} D\left(A_{1}, A_{2}, \ldots, A_{k}\right),{ }_{l}^{2} D\left(A_{1}, A_{2}, \ldots, A_{k}\right)$ accelerate $\delta_{1} \cup \mathcal{\delta}_{2} \cup \cdots \cup \mathcal{\delta}_{k}$.

LEMMA. Let $\left(S_{n}\right)$ be a convergent sequence with limit $S$. Let $\left(A_{1}^{(n)}\right)$, $\left(A_{2}^{(n)}\right), \ldots,\left(A_{k}^{(n)}\right)$ be $k$ sequences which accelerate the convergence of $\left(S_{n}\right)$. Let $i$ : $\mathbf{N} \rightarrow\{1,2, \ldots, k\}$. Then the sequence $\left(A_{i(n)}^{(n)}\right)$ accelerates the convergence of $\left(S_{n}\right)$.

Proof. Let $\varepsilon \in \mathbf{R}^{+*}$. For each $i \in\{1,2, \ldots, k\}$, there is $m_{i} \in \mathbf{N}$ such that $\forall m>m_{i}: d\left(A_{i}^{(m)}, S\right) / d\left(S_{m}, S\right)<\varepsilon$. Letting $M=\max \left\{m_{i} \mid i \in\{1,2, \ldots, k\}\right\}$, we obtain

$$
\forall m>M: d\left(A_{i(m)}^{m}, S\right) / d\left(S_{m}, S\right)<\varepsilon .
$$

Remark. The lemma is not true with $\Delta$-acceleration. Here is a counterexample: $S_{n}=1 / n, A_{1}^{(n)}=1 / n^{2}, A_{2}^{(n)}=1 / 2 n^{2}, i(n)=\left(3+(-1)^{n}\right) / 2$. We have

$$
\begin{aligned}
& \lim _{n \rightarrow \infty} d\left(A_{1}^{(n+1)}, A_{1}^{(n)}\right) / d\left(S_{n+1}, S_{n}\right)=0, \\
& \lim _{n \rightarrow \infty} d\left(A_{2}^{(n+1)}, A_{2}^{(n)}\right) / d\left(S_{n+1}, S_{n}\right)=0, \\
& \lim _{n \rightarrow \infty} d\left(A_{i(n+1)}^{(n+1)}, A_{i(n)}^{(n)}\right) / d\left(S_{n+1}, S_{n}\right)=1 / 2 .
\end{aligned}
$$

Proof of Theorem 2 . We indicate a proof only for ${ }_{1}^{0} D\left(A_{1}, A_{2}, \ldots, A_{k}\right)$ denoted by $A$. One can easily adapt this proof for the other cases. Let $\left(S_{n}\right) \in \mathcal{S}_{1} \cup \mathcal{S}_{2}$ $\cup \cdots \cup \delta_{k}$. Let $S$ be its limit. Let $I_{0}$ be the set of integers $i \in\{1,2, \ldots, k\}$ such that $A_{i}$ accelerates and $\Delta$-accelerates $\left(S_{n}\right)$. From the assumptions $I_{0}$ is nonempty. If $j \notin I_{0}$, there exists $\varepsilon_{j} \in \mathbf{R}^{\dagger *}$ and $n_{j} \in \mathbf{N}$ such that

$$
\forall n>n_{j}, \quad d\left(A_{j}^{(n+1)}, A_{j}^{(n)}\right) / d\left(S_{n+1}, S_{n}\right)>\varepsilon_{j} .
$$

We write $\varepsilon=\min \left\{\varepsilon_{j} \mid j \notin I_{0}\right\}, N=\max \left\{n_{j} \mid j \notin I_{0}\right\}$; we obtain

$$
\forall n \geqslant N, \forall j \notin I_{0}: d\left(A_{j}^{(n+1)}, A_{j}^{(n)}\right) / d\left(S_{n+1}, S_{n}\right)>\varepsilon .
$$

Similarly there exists $N^{\prime} \geqslant N$ such that

$$
\forall n \geqslant N^{\prime}, \forall i \in I_{0}: d\left(A_{i}^{(N+1)}, A_{i}^{(n)}\right) / d\left(S_{n+1}, S_{n}\right)<\varepsilon .
$$

This implies that, for each $n \geqslant N^{\prime}+l, i(n) \in I_{0}$. From the lemma we can conclude

$$
\lim _{n \rightarrow \infty} d\left(A_{i(n)}^{(n)}, S\right) / d\left(S_{n}, S\right)=0 .
$$

Remark 1. The assumptions of Theorem 2 are often satisfied in practice, but generally it is difficult to show that they are satisfied for specified transformations $A_{i}$ and large $\delta_{i}$. Nevertheless, this theorem justifies and explains the efficiency of the selection methods ${ }_{l} D$ as we shall see in the practical cases of Section 3.

Remark 2. We can generalize the notion of fair transformations; we say that the sequence transformation $A$ is $h$-fair $(h \in N)$ for $\mathcal{S}$ if, for every sequence $\left(S_{n}\right) \in \mathcal{S}$,

$\left({ }_{h} F_{1}\right)$ either $A$ accelerates and $\Delta$-accelerates $\left(S_{n}\right)$,

$\left({ }_{h} F_{2}\right)$ or

$$
\exists \varepsilon>0, \exists n_{0} \in \mathbf{N} \forall n \geqslant n_{0} \max _{0<r<h} d\left(A^{(n-r+1)}, A^{(n-r)}\right) / d\left(S_{n+1}, S_{n}\right)>\varepsilon .
$$


We obtain [ $A$ fair] $\Leftrightarrow\left[A\right.$-fair]. If $h>h^{\prime},\left[A h^{\prime}\right.$-fair] $\Rightarrow[A h$-fair]. The assumption " $A h$-fair" is more often true than the assumption " $A$ fair". However, Theorem 2 with $l>h$ is still true.

3. Computational Experiences. The computational experiences presented here were made with C. Brezinski's codes [4], and I would like to take this opportunity to thank him, for his excellent advice and support.

Example 1. In Table 1 we present the first 10 steps for the $\delta$ transformations in competition (see Section 1, Example 3) when they are applied to the sequence

$$
S_{n}=\frac{1}{2}\left(\frac{1}{2}\right)^{2}+\frac{1}{4}\left(\frac{1 \cdot 3}{2 \cdot 4}\right)^{2}+\cdots+\frac{1}{2 n}\left(\frac{1 \cdot 3 \cdots(2 n-1)}{2 \cdot 4 \cdots(2 n)}\right)^{2} \rightarrow .22005074 \ldots .
$$

The selected transformation by the method ${ }^{\circ} D$ at the $n$th step is indicated with an $X$. We see that this chosen transformation is always among the transformations $R$, $\rho$, or $\theta$, which are here the transformations which accelerate the convergence. Consequently, we can say that the choice is correct. The method ${ }^{1} D$ is also correct; at the step $n$ with $n \geqslant 3$, the chosen transformation is always the $\rho$-algorithm. The method ${ }^{2} D$ gives exactly the same results that the method ${ }^{0} D$.

\section{TABLE 1}

\begin{tabular}{|c|c|c|c|c|c|c|}
\hline$S_{n}$ & $R(1 / n)$ & $\varepsilon$ & $p(n)$ & Ov. & $\theta$ & $\Delta^{2}$ it. \\
\hline .125 & 1 & 5 & 1 & 1 & $\begin{array}{r}25 \\
\end{array}$ & $\begin{array}{r}25 \\
1\end{array}$ \\
\hline 16015625 & .19531250 & $\begin{array}{c}16015625 \\
2 \\
\end{array}$ & $\begin{array}{c}.16015625 \\
2 \\
\end{array}$ & $\begin{array}{c}16015625 \\
2 \\
\end{array}$ & $\begin{array}{c}.16015625 \\
2 \\
\end{array}$ & $\begin{array}{c}.16015625 \\
2 \\
\end{array}$ \\
\hline .1764 & $\begin{array}{c}.2158 ? 031 \\
2\end{array}$ & $\begin{array}{c}19046336 \\
3 \\
\end{array}$ & $\begin{array}{c}22077047 \\
1 \\
\end{array}$ & $\begin{array}{c}19046336 \\
5 \\
\end{array}$ & .176 & $\begin{array}{c}19046336 \\
3 \\
\end{array}$ \\
\hline 330 & $\begin{array}{c}21959093 \\
2\end{array}$ & $\begin{array}{c}-19838255 \\
5\end{array}$ & $\begin{array}{c}22033281 \\
(1) \\
8\end{array}$ & $\begin{array}{c}20227823 \\
4\end{array}$ & $\begin{array}{c}-21918327 \\
3\end{array}$ & $\begin{array}{c}-19838255 \\
5\end{array}$ \\
\hline 51 & $\begin{array}{c}.22002856 \\
2\end{array}$ & $\begin{array}{c}20570155 \\
6\end{array}$ & $\begin{array}{c}22004511 \\
(1)\end{array}$ & $\begin{array}{c}20782237 \\
5 \\
\end{array}$ & $\begin{array}{c}.21966574 \\
3\end{array}$ & $\begin{array}{c}20936274 \\
4\end{array}$ \\
\hline 26 & $\begin{array}{c}.22005258 \\
2 \\
x\end{array}$ & $\begin{array}{c}20871905 \\
6\end{array}$ & $\begin{array}{c}X \\
.22004902 \\
(1)\end{array}$ & $\begin{array}{c}.21095162 \\
5\end{array}$ & $\begin{array}{c}.21984779 \\
3\end{array}$ & $\begin{array}{c}.21158726 \\
4 \\
\end{array}$ \\
\hline 19920946 & $\begin{array}{c}X \\
.22005113 \\
(3)\end{array}$ & $\begin{array}{c}.21158833 \\
6\end{array}$ & $\begin{array}{c}.22005079 \\
2\end{array}$ & $\begin{array}{c}.21293141 \\
5\end{array}$ & $\begin{array}{c}.22005077 \\
1\end{array}$ & $\begin{array}{c}.21590176 \\
4\end{array}$ \\
\hline 30 & $\begin{array}{c}22005076 \\
1\end{array}$ & $\begin{array}{c}.21305538 \\
6\end{array}$ & $\begin{array}{c}.22005076 \\
1 \\
x\end{array}$ & $\begin{array}{c}.21428050 \\
5\end{array}$ & $\begin{array}{c}\mathrm{X} \\
.22005078 \\
(3) \\
\end{array}$ & $\begin{array}{c}.21659597 \\
4 \\
\end{array}$ \\
\hline .20353087 & $\begin{array}{c}.22005074 \\
1\end{array}$ & $\begin{array}{c}.21447121 \\
6 \\
\end{array}$ & $\begin{array}{c}X \\
.22005074 \\
(1) \\
y\end{array}$ & $\begin{array}{c}.21524960 \\
5 \\
\end{array}$ & $\begin{array}{c}.22005077 \\
3 \\
\end{array}$ & $\begin{array}{c}.21839937 \\
4\end{array}$ \\
\hline .20508314 & $\begin{array}{c}22005074 \\
1\end{array}$ & $\begin{array}{c}.21529441 \\
6\end{array}$ & $\begin{array}{c}22005074 \\
(1)\end{array}$ & $\begin{array}{c}21597391 \\
5\end{array}$ & $\begin{array}{c}22005078 \\
3\end{array}$ & $\begin{array}{c}21861073 \\
4\end{array}$ \\
\hline
\end{tabular}

Since we know that $S=.22005074$, we can determine, at each step, the exact rank of each transformation (this rank is indicated in Table 1 by a number under each transformed point). For example at step 4 (the first significant one) the $\rho$-algorithm gives .22033281 and this is the best transformed point; the rank of the $\rho$-algorithm at step 4 is consequently 1 . The method ${ }^{0} D$ chooses the $\rho$-algorithm at step 4 ; this is the best possible choice. At steps 5 and 6 the choice is still the best possible, but at 
step $7{ }^{\circ} \mathrm{D}$ chooses the Richardson process whose rank is 3 . The rank sequence of chosen transformations is $(1,1,1,3,3,1,1)$. This is not the best possible rank sequence, which is $(1,1,1,1,1,1,1)$. However, it is a good rank sequence because all ranks are $\leqslant 3$, and there are only three sequence transformations accelerating the convergence of $\left(S_{n}\right)$. For the two following examples, we have only indicated the rank sequence of the chosen transformation when the method ${ }^{0} D$ is applied.

Example 2. $S_{n}=\exp (-\sqrt{n} / 10 \sqrt{2}) / n$.

TABLE 2

\begin{tabular}{|r|r|r|r|r|r|r|r|r|r|r|r|r|r|r|r|r|r|r|r|r|r|r|r|r|}
\hline 0 & 1 & 2 & 3 & 4 & 5 & 6 & 7 & 8 & 9 & 10 & 11 & 12 & 13 & 14 & 15 & 16 & 17 & 18 & 19 & 20 & 21 & 22 & 23 & 24 \\
\hline & & & 2 & 1 & 1 & 3 & 3 & 1 & 1 & 1 & 1 & 1 & 2 & 2 & 3 & 3 & 3 & 3 & 3 & 3 & 3 & 3 & 2 & 2 \\
\hline 25 & 26 & 27 & 28 & 29 & 30 & 31 & 32 & 33 & 34 & 35 & 36 & 37 & 38 & 39 & 40 & 41 & 42 & 43 & 44 & 45 & 46 & 47 & 48 & 49 \\
\hline 3 & 3 & 2 & 3 & 3 & 1 & 1 & 1 & 4 & 2 & 3 & 3 & 2 & 1 & 3 & 1 & 2 & 2 & 2 & 2 & 1 & 2 & 2 & 2 & 2 \\
\hline
\end{tabular}

Only three transformations accelerate $\left(S_{n}\right): \varepsilon$-algorithm, $\rho$-algorithm, and $\Delta^{2}$ iterated. When $n$ is large enough, at each step $n,{ }^{0} D$ chooses one of these three transformations.

Example 3. $S_{2 n}=(1 / 2 n), S_{2 n+1}=(4 n+5) /(2 n+2)^{2}$.

TABLE 3

\begin{tabular}{|r|r|r|r|r|r|r|r|r|r|r|r|r|r|r|r|r|r|r|r|r|r|r|r|r|}
\hline 0 & 1 & 2 & 3 & 4 & 5 & 6 & 7 & 8 & 9 & 10 & 11 & 12 & 13 & 14 & 15 & 16 & 17 & 18 & 19 & 20 & 21 & 22 & 23 & 24 \\
\hline & & & 5 & 2 & 2 & 2 & 3 & 3 & 4 & 4 & 3 & 3 & 3 & 3 & 3 & 3 & 3 & 3 & 4 & 3 & 2 & 3 & 2 & 3 \\
\hline 25 & 26 & 27 & 28 & 29 & 30 & 31 & 32 & 33 & 34 & 35 & 36 & 37 & 38 & 39 & 40 & 41 & 42 & 43 & 44 & 45 & 46 & 47 & 48 & 49 \\
\hline 2 & 2 & 2 & 2 & 2 & 2 & 2 & 2 & 2 & 2 & 2 & 2 & 2 & 2 & 2 & 2 & 2 & 2 & 2 & 2 & 2 & 2 & 2 & 2 & 2 \\
\hline
\end{tabular}

Only two transformations accelerate $\left(S_{n}\right): \varepsilon$-algorithm and $\rho$-algorithm. The best one $(\varepsilon$-alg.) is not chosen because the transformed sequence is alternating; when $n$ is large enough, the choice at the step $n$ is the $\rho$-algorithm.

Université des Sciences et Techniques de Lille

I UER D'IEEA

59655 Villeneuve D’Ascq Cedex, France

1. C. BrezinsKa, "Accélération de suites à convergence logarithmique," C. R. Acad. Sci. Paris Sér. $A-B$, v. 273, 1971, pp. 727-730.

2. C. BreznNSKI, "Etudes sur les $\varepsilon$ et $\rho$-algorithmes," Numer. Math., v. 17, 1971, pp. 153-162.

3. C. Brezinski, Accélération de la Convergence en Analyse Numérique, Locture Notes in Math., vol. 584, Springer-Verlag, Heidelberg, 1977. 1978.

4. C. Brezinski, Algorithmes d'Accélération de la Convergence: Etude Numérique, Technip, Paris,

5. C. Brezinski, A General Extrapolation Algorithm, Publ. A. N. O. No. 9, Univ. des. Sci. et Tech. de Lille, 1979.

6. F. Cordellier, Sur la Régularité des Procédés $\delta^{2} d^{\prime}$ 'Aitken et $W$ de Lubkin, Publ. A. N. O. No. 10, Univ. des Sci. et Tech. de Lille, 1979.

7. J. P. Delahnye, Etude Générale des Procédés de Choix Entre Transformations de Suites en Accélération de la Convergence, Publ. A. N. O., Univ. des Sci. et Tech. de Lille. (To appear.)

8. P. J. LAURent, Etude des procédés d'Extrapolation en Analyse Numérique, Thèse, Grenoble, 1964.

9. K. J. OVerholt, “Extended Aitken acceleration,” BIT, v. 6, 1965, pp. 122-132.

10. D. Shanks, "Non linear transformations of divergent and slowly convergent series," J. Math. Phys., v. 34, 1955, pp. 1-42.

11. P. WYNN, "On a device for computing the $e_{m}\left(S_{n}\right)$ transformation," MTAC, v. 10, 1956, pp. 91-96. 